\title{
Electronic structures in $\mathrm{VO}_{2}$ using the periodic polarizable point-ion shell model and DV-X $\alpha$ method
}

\author{
H. Nakatsugawa and E. Iguchi* \\ Materials Science, Department of Mechanical Engineering and Materials Science, Faculty of Engineering, \\ Yokohama National University, Tokiwadai, Hodogaya-Ku, Yokohama, 240 Japan
}

(Received 7 August 1996; revised manuscript 25 September 1996)

\begin{abstract}
Electronic structures in the metallic $\mathrm{VO}_{2}$ phase above the metal-insulator (MI) transition temperature of $T_{c}$ and the insulating phase below $T_{c}$ have been investigated using the combination of the three-dimensional periodic shell model and the discrete-variational (DV)-X $\alpha$ cluster method. Besides the correlation effect for $d_{\|}$ electrons, the Hamiltonian in the insulating phase includes the Anderson's attractive potential due to the electron-phonon interactions which stabilize the three-dimensional periodic distribution of $\mathrm{V}^{4+}-\mathrm{V}^{4+}$ dimers. The shell model estimates the electron-phonon coupling constant and provides direct theoretical evidence that the dimers are stable in the low-temperature phase. The DV-X $\alpha$ cluster method calculates the electron energies in $\left[\mathrm{V}_{2} \mathrm{O}_{10}\right]^{-12}$ clusters and the value for the intersite repulsive nearest-neighbor $d$ - $d$ Coulombic interaction which quantifies the correlation effect for $d_{\|}$electrons. The electron-phonon interaction effect and the correlation effect for $d_{\|}$electrons are found to split $d$ band into the empty upper and the occupied lower Hubbard bands and also to result in an obvious energy gap between these bands in the insulating phase. In the metallic phase, the nonresolved $d$ band overlaps the $\pi^{*}$ band and they construct a partially filled conduction band. These calculations explain well the $\mathrm{MI}$ transition in $\mathrm{VO}_{2}$ and, in particular, the electron-phonon interaction assessed by the periodic shell model is an indispensable contribution in the stabilization of the insulating phase. [S0163-1829(97)01003-5]
\end{abstract}

\section{INTRODUCTION}

When temperature decreases, $\mathrm{VO}_{2}$ undergoes a crystallographic phase transition at $T_{c}=340 \mathrm{~K}$. The paramagnetic metal phase above $T_{c}$ has a rutile-type crystal structure, while the lattice is distorted to a monoclinic crystal structure in the nonmagnetic insulating phase below $T_{c}$ (Refs. 1-3). Thus this phase transition is a metal-insulator (MI) transition. The insulating $\mathrm{VO}_{2}$ phase contains the three-dimensional periodic distribution of $\mathrm{V}^{4+}-\mathrm{V}^{4+}$ dimers formed by the assist of electron-phonon interactions. ${ }^{4}$ Since $\mathrm{VO}_{2}$ contains only a $\mathrm{V}^{4+}\left(3 d^{1}\right)$ electron configuration within the dimers below $T_{c}$, the type of optical transition is inevitably an intrapair transition in the insulating phase. ${ }^{5}$ Then, the electrons in $\mathrm{V}^{4+}$ ions are expected to excite locally from a ground state of the pair to a higher-energy state. Goodenough ${ }^{6}$ explained the MI transition in $\mathrm{VO}_{2}$ within the framework of a simple crystalfield theory based upon the Mott-Hubbard regime. Above $T_{c}$, the $\pi^{*}$ band overlaps the half-filled $d_{\|}$band. When temperature decreases below $T_{c}, \pi^{*}$ rises in energy beyond the Fermi level and becomes empty whereas the $d_{\|}$splits into the empty band and the occupied one which should be narrow. ${ }^{6}$

The vacuum-ultraviolet photoemission spectra (UPS) ${ }^{7}$ on $\mathrm{VO}_{2}$ showed the disappearance of the states at the Fermi level when going from the high temperature to the lowtemperature phase. Shin et al. pointed out that the correlation effect for $d_{\|}$electrons became more important when $d_{\|}$band splitted into two bands. ${ }^{7}$ At temperatures below $T_{c}$, the $\mathrm{O} 1 s$ soft-X-ray-absorption spectra show a clear splitting of about $1 \mathrm{eV}$ between the unoccupied $d_{\|}$band and $\pi^{*}$ band above the Fermi level. ${ }^{8}$ This result is in favor of the theory which explains the MI transition in terms of localized pairing interac- tions within $\mathrm{V}^{4+}-\mathrm{V}^{4+}$ dimers. ${ }^{6}$ These results indicate that not only the correlation effect for $d_{\|}$electrons but also the electron-phonon interaction effect participate directly in the MI transition. Therefore, this material requires more detailed theoretical works which can distinguish the effect of the lattice distortion induced by the electron-phonon interactions from that of electron localization so as to clarify the origin of the MI transition.

The electronic structures in $\mathrm{VO}_{2}$ were studied using various band calculations ${ }^{9-11}$ and cluster calculations. ${ }^{12,13} \mathrm{Re}-$ cently, the discrete-variational (DV)-X $\alpha$ cluster method ${ }^{14-20}$ has been applied to several ionic crystals so as to investigate the electronic structures without the muffin-tin potential approximation which is used in the multiple-scattering (MS)$X \alpha$ method $^{21}$ and so on. The primary aim in the present report is to interpret the MI transition theoretically using the method somewhat different from the previous literatures. $^{9-13,22}$ To this end, the Hamiltonian involving the electron-phonon interactions has to be described properly.

Anderson ${ }^{23,24}$ has shown that a cation-cation pairing phenomenon is greatly facilitated through local bond distortion, as if a negative effective pairwise interaction is operative on a bond site. The resultant pair is stabilized by the lattice distortion mainly due to the electron-phonon interaction effect. ${ }^{25}$ In the insulating $\mathrm{VO}_{2}$ phase, one can write in the spirit of the Anderson Hamiltonian as follows: ${ }^{4,23}$

$$
\begin{aligned}
H_{\mathrm{eff}}= & \sum_{i \sigma} \epsilon_{i} n_{i \sigma}+U \sum_{i \neq j} n_{i \uparrow} n_{j \downarrow}+\frac{1}{2} c \sum_{i \neq j} x_{i j}^{2} \\
& -\frac{\lambda}{2} \sum_{i \neq j} x_{i j}\left(n_{i \uparrow}+n_{j \downarrow}\right),
\end{aligned}
$$


here $i$ and $j$ are nearest-neighbor sites, $\epsilon_{i}$ is an atomic energy at the $i$ th site, $U$ represents an intersite repulsive nearestneighbor $d$ - $d$ Coulombic interaction, $c$ is a relevant elastic constant, $\lambda$ is an electron-phonon coupling constant between $\mathrm{V}^{4+}$ ions on bond sites, and $x_{i j}$ is the change in the spacing between the ions on formation of pairs. Since $i$ and $j$ refer to the sites of the cations on bond sites, $H_{\text {eff }}$ is expected to operate only in the electrons of the $d_{\|}$occupied narrow band in the insulating phase. The third term in the right-hand side of Eq. (1) is denoted as $E_{D}$ which represents an increase in the lattice energy due to the local lattice distortion induced by the formation of $\mathrm{V}^{4+}-\mathrm{V}^{4+}$ dimers and the fourth term is the electron-phonon interaction energy abbreviated by $E_{e \text {-ph }}$. The sum of these two terms is the Anderson's attractive potential, ${ }^{23,24}$ i.e., $V=\left[c \sum x_{i j}^{2}-\lambda \sum x_{i j}\left(n_{i \uparrow}+n_{j \downarrow}\right)\right] / 2$. When $n_{i \uparrow}=1$ and $n_{j \downarrow}=1$, the $\mathrm{V}^{4+}-\mathrm{V}^{4+}$ pairs are stabilized by the assist of the electron-phonon interaction and then form dimers. The optimum condition ${ }^{23}$ for the Anderson's attractive potential, i.e., $\partial V / \partial x_{i j}=0$, yields $V=-\lambda^{2} / 2 c$. Then the eigenvalue of Eq. (1) is expressed as

$$
E=2 \epsilon_{i}+U-\frac{\lambda^{2}}{2 c}
$$

where the second and the third terms in the right-hand side indicate the electron correlation effect and the electronphonon interaction effect, respectively.

The most important point in Eq. (2) is the last term which implies that the $d_{\|}$occupied band, i.e., the lower Hubbard band, lowers in energy by $\lambda^{2} / 2 c$. This is consistent with the idea of Mott et $a l^{26,27}$ that the gap between the upper and the lower Hubbard bands increases by pairing. As shown in Eq. (1), the estimate of the magnitude for $\lambda^{2} / 2 c$ requires the calculation of the change in the lattice energy induced by pairing. The very small lattice distortion around the pairs imposes the stringent constraints for the required accuracy in making the theoretical estimates. The polarizable point-ion shell model meets surely this requirement because Dienes et $a l .{ }^{28}$ constructed this model for the purpose of calculating a change in a lattice energy due to slight ionic displacement induced by an imperfection in an insulating ionic crystal. Though their shell model is impracticable in $\mathrm{VO}_{2}$ because of the three-dimensional periodic distribution of $\mathrm{V}^{4+}-\mathrm{V}^{4+}$ dimers, our previous report ${ }^{29}$ developed this model to the three-dimensional periodic shell model which can be applied to ionic crystals containing three-dimensional distributions of imperfections even after relaxation takes place like the $\mathrm{Ti}_{n} \mathrm{O}_{2 n-1}$ homologous series. ${ }^{30-33}$ The calculation on the two-dimensional periodic surface structure of $\mathrm{NiO}$ rationalizes our periodic shell model. ${ }^{29}$ The shell model calculations including the Anderson's attractive potential have been repeated in our previous reports which treat the stability of small polarons. ${ }^{33-38}$

Since the DV- $X \alpha$ cluster method can calculate the energies of electron states, ${ }^{14-20}$ the combination of the periodic shell model and the cluster method must contain the possibility to clarify the origin of the $\mathrm{MI}$ transition in $\mathrm{VO}_{2}$ from the energetic point of view. Therefore, the calculation using this combination is of great significance in order to understand the very important phenomena of $\mathrm{VO}_{2}$, particularly the MI transition.

\section{THEORETICAL PROCEDURES}

\section{A. Crystal structure of $\mathrm{VO}_{2}$}

The high-temperature metallic $\mathrm{VO}_{2}$ phase has the tetragonal rutile structure $\left(\mathrm{P}_{2} / \mathrm{mnm}\right)$, each vanadium ion being located at the center of an oxygen octahedron. The rutile structure has the following primitive translation vectors: ${ }^{1}$

$$
\mathbf{a}_{r}=a_{r} \mathbf{i}, \quad \mathbf{b}_{r}=a_{r} \mathbf{j}, \quad \mathbf{c}_{r}=c_{r} \mathbf{k},
$$

where $a_{r}=4.530 \AA$ and $c_{r}=2.869 \AA$. The subscript $r$ refers to the rutile structure, and $\mathbf{i}, \mathbf{j}$, and $\mathbf{k}$ are the usual rectangular unit vectors. Each unit cell contains two $\mathrm{V}^{4+}$ ions $(0,0,0$ and $\left.\frac{1}{2}, \frac{1}{2}, \frac{1}{2}\right)$ and four $\mathrm{O}^{2-}$ ions $(u, u, 0$ and so on). In particular, the high-temperature phase is characterized by chains of equidistant $\mathrm{V}^{4+}$ ions along the rutile $c_{r}$ axis.

On the other hand, the low-temperature insulating phase has the monoclinic structure $\left(P 2_{1} / c\right)$. The lattice parameters are $|\mathbf{a}|=5.743 \AA,|\mathbf{b}|=4.517 \AA,|\mathbf{c}|=5.375 \AA$, and $\beta=122.61^{\circ}$ where $\mathbf{a}, \mathbf{b}$, and $\mathbf{c}$ are the primitive translation vectors of the monoclinic structure, and $\beta$ is the angle between a and $\mathbf{c}^{2,3}$ To within experimental accuracy, there are the following relations existing between the rutile-type unit cell and the monoclinic one of $\mathrm{VO}_{2}$ (Ref. 3):

$$
\mathbf{a} \simeq 2 \mathbf{c}_{r}, \quad \mathbf{b} \simeq \mathbf{a}_{r}, \quad \mathbf{c} \simeq \mathbf{b}_{r}-\mathbf{c}_{r} .
$$

Thus the monoclinic structure contains four " $\mathrm{VO}_{2}$ " molecules per unit cell. A striking feature of the monoclinic phase is the presence of cation-cation pairs along the $a$ axis, alternate $\mathrm{V}^{4+}-\mathrm{V}^{4+}$ separations form a zig-zag chain, i.e., $3.1246 \AA$ and $2.6541 \AA$, that is, the cation-cation bondings form the dimers.

It should be however noticed that the crystal structure and the lattice parameters in the insulating phase are the ones obtained in the $\mathrm{x}$-ray analyses ${ }^{1-3}$ after the $\mathrm{V}^{4+}-\mathrm{V}^{4+}$ dimers are formed with the assistance of the electron-phonon interactions and that the ionic displacement due to these dimers is induced on each ion. The present report intends to investigate into the formation kinetics of the dimers, the relaxation kinetics of the lattice, and finally the calculations of the electron states in the ensuing crystallographic structure which is the one observed actually in the insulating phase. This implies that the initial ionic distribution transfers to the final one through the formation of dimers and the relaxation processes. Though the final structure is known in the experiments, the initial one is unknown. Then the theoretical treatment requires a hypothetical crystal structure in the initial state. To this end, the ionic positions in the initial state of the insulating phase represented in the units of $\mathbf{a}, \mathbf{b}$, and $\mathbf{c}$ are assumed as

$$
\begin{aligned}
& \mathrm{V}^{4+}: \quad x=x_{0}, \quad y=y_{0}, \quad z=z_{0}, \\
& \mathrm{O}_{1}{ }^{2-}: \quad x=0.25-0.5 u, \quad y=0.5-u, \quad z=0.5-u \text {, } \\
& \mathrm{O}_{11}^{2-}: \quad x=0.25+0.5 u, \quad y=1.0-u, \quad z=u \text {. }
\end{aligned}
$$

We have employed $u=0.305$, i.e., the rutile parameter in $\mathrm{VO}_{2}$ (Ref. 1). The ionic positions of other three " $\mathrm{VO}_{2}$ " molecules are identified by the crystallographic treatment proposed by Anderson. ${ }^{3}$

When temperature decreases below $T_{c}, \mathrm{~V}^{4+}$ moves from the position in the rutile structure of the metallic phase mainly due to soft-phonon modes at the $R$ point of the primi- 
TABLE I. The parameters of $\mathrm{VO}_{2}$ employed in the shell model calculations: free-ion polarizabilities $\alpha^{0}$; electronic polarizabilities $\alpha^{e}$; average exciting energies $E_{\mathrm{ex}}$; and the shell parameters $Q$.

\begin{tabular}{lcccc}
\hline \hline Ion & $\alpha^{0}\left(\AA^{3}\right)$ & $\alpha^{e}\left(\AA^{3}\right)$ & $E_{\text {ex }}(\mathrm{eV})$ & $Q(e)$ \\
\hline $\mathrm{V}^{4+}$ & 0.123 & 0.291 & 130.19 & 7.691 \\
$\mathrm{O}^{2-}$ & 3.880 & 2.636 & 8.00 & 1.978 \\
\hline \hline
\end{tabular}

tive tetragonal Brillouin zone, ${ }^{39}$ whereas $\mathrm{O}^{2-}$ ions are nearly fixed even after the phase transition takes place. Therefore, the oxygen positions in Eq. (5) are essentially the ones in the rutile structure. The initial positions of $\mathrm{V}^{4+}$ ions, $\left(x_{0}, y_{0}, z_{0}\right)$, in the insulating phase were determined in the following way. The relaxation due to the electric field acting on each ion and another relaxation due to the electron-phonon interactions displace $\mathrm{V}^{4+}$ ions. Consequently the dimers are formed and the $\mathrm{V}^{4+}-\mathrm{V}^{4+}$ spacings separate into two groups. The relaxation calculation using the three-dimensional pointion shell model ${ }^{29}$ was iterated, by changing the initial positions of $\mathrm{V}^{4+}$ ions, until the final positions yield the experimental separations, i.e., $3.1246 \AA$ and $2.6541 \AA$. In the end, $\left(x_{0}, y_{0}, z_{0}\right)=(0.2434,09754,0.0244)$. The $\mathrm{V}^{4+}-\mathrm{V}^{4+}$ separation is $2.6697 \AA$ in the initial state and $2.6541 \AA$ in the final state.

Calculations on the metallic phase were based upon the rutile structure. If represented in the units of the monoclinic translation vectors, $\mathbf{a}, \mathbf{b}$, and $\mathbf{c}$, the position of $\mathrm{V}^{4+}$ is $\left(x_{0}, y_{0}\right.$, $\left.z_{0}\right)=(1 / 4,0.0,0.0)$, while the oxygen positions are already indicated in Eq. (5). The atomic displacement was not taken into account in the metallic phase because of the two reasons; (i) the shell model is applicable only in insulating ionic crystals, ${ }^{28-38}$ and (ii) the displacement on each atom in the metallic phase is remarkably small even if the relaxation is calculated by the shell model.

\section{B. Shell model and electron-phonon interactions}

Though $\mathrm{V}^{4+}-\mathrm{V}^{4+}$ dimers induced by the electron-phonon interactions stabilize the insulating phase, ${ }^{4}$ the lattice is slightly distorted because the ionic interaction modulated by the periodic distribution of the dimers displaces each ion. Then, the specific test in the stability of the insulating phase requires the calculation based upon the three-dimensional periodic polarizable point-ion shell model. A brief description on the framework of this model will be given because full details were published elsewhere. ${ }^{29-38}$

The lattice energy in a rigid and unpolarized crystal is given by the sum of the long-range Madelung energy $\left(E_{M}\right),{ }^{40,41}$ the short-range repulsive energy $\left(E_{R}\right)$, and the van der Waals energy $\left(E_{\mathrm{vdW}}\right)$. The Born-Mayer constants and the van der Waals constants included in the lattice energy were obtained using the wave functions, the electronic polarizabilities, $\alpha^{e}$, and the average exciting energies, $E_{\mathrm{ex}}$, of ions calculated by the theoretical procedures ${ }^{42-49}$ which were employed in our previous reports. ${ }^{29-38,50-53}$ The values for $\alpha^{e}$ and $E_{\text {ex }}$ are collected in Table I together with free-ion polarizabilities of Pauling, ${ }^{54} \alpha^{0}$, and the shell parameters, $Q$. The Born-Mayer constants of the ion pairs involving $\mathrm{V}^{4+}$ ions require the modification from those involving $\mathrm{V}^{5+}$ ions based upon the scaling procedure using the ionic radii. ${ }^{55}$ (A table of the Born-Mayer and the van der Waals constants will be furnished by the authors upon request.)

When regular arrays of imperfections are formed in a rigid and unpolarized crystal, each ion is displaced so as to minimize the energy of the crystal, that is, a relaxation takes place. The displacement of an ion results in a dipole moment on that ion. The total energy of the crystal, $\left(E_{T}\right)_{a}$, is then given by

$$
\left(E_{T}\right)_{a}=E_{L}+\left(E_{\text {Relax }}\right)_{a},
$$

where the subscript $a$ indicates the energy after the relaxation takes place, and $\left(E_{\text {Relax }}\right)_{a}$ represents the relaxation energy which consists of the changes in the Madelung, the repulsive, the van der Waals energies, $\Delta E_{M}, \Delta E_{R}, \Delta E_{\mathrm{vdW}}$, and the polarization energy, $E_{p}$ (see Refs. 28 and 29).

After the relaxation has taken place, the vector between ions, $i$ and $j$, changes from $\mathbf{R}_{i j}$ to the effective one $\mathbf{R}_{i j}^{a}$ :

$$
\mathbf{R}_{i j}^{a}=\mathbf{R}_{i j}+\frac{\boldsymbol{\mu}_{i}^{a}}{Q_{i}}-\frac{\boldsymbol{\mu}_{j}^{a}}{Q_{j}},
$$

where $\boldsymbol{\mu}_{i}^{a}$ and $Q_{i}$ are the dipole moment and the shell parameter of the $i$ th ion. The shell parameters were determined by the method developed from the theory of Dick and Overhauser ${ }^{56}$ by Shanker and Gupta. ${ }^{57,58}$ In this scheme, the total energy $\left(E_{T}\right)_{a}$ is a function of dipole moments. The equilibrium dipole moments are obtained by minimizing the total energy with respect to the components of individual dipole moments as follows: ${ }^{28-38}$

$$
\frac{\partial\left(E_{T}\right)_{a}}{\partial \mu_{i k}^{a}}=0, \quad k=x, \quad y, \text { or } z,
$$

where $\mu_{i k}^{a}$ is the $k$ component of the $i$ th dipole moment. A unit cell in the insulating phase contains twelve ions, i.e., four $\mathrm{V}^{4+}$ ions and eight $\mathrm{O}^{2-}$ ions. Since the threedimensional translation operation yields the same magnitude for the dipole moment on the $i$ th ion in every unit cell, the number of unknowns is $3 \times 12$, i.e., $\mu_{i x}, \mu_{i y}$, and $\mu_{i z}(i=1-$ 12) and the matrix technique solves each component of the dipole moments. ${ }^{29}$

Taking into account of the Anderson's idea, ${ }^{2,24}$ the Hamiltonian involving the electron-phonon interaction effect and the lattice distortion due to this interaction has the form of Eq. (1) with the eigenvalue in Eq. (2). The total energy of the crystal containing ordered arrays of the dimers is given as

$$
\left(E_{T}\right)_{b}=E_{L}+\left(E_{\text {Relax }}\right)_{b}+E_{e-\mathrm{ph}},
$$

where the subscript $b$ indicates the energies after the electron-phonon interactions are considered and the last term, $E_{e \text {-ph }}$, represents the electron-phonon interaction energy corresponding to the fourth term of the right-hand side in Eq. (1), i.e., $E_{e \text {-ph }}=-\lambda \Sigma x_{i j}$. The description on the equilibrium equation involving the electron-phonon interactions was repeated in our previous literatures. ${ }^{34-38}$ Each component of the dipole moments can be obtained also by the matrix technique. Therefore, the following relations estimate the magnitudes for $\lambda$ and $c$ :

$$
-\frac{\lambda^{2}}{2 c}=\left(E_{T}\right)_{b}-\left(E_{T}\right)_{a}=E_{D}+E_{e-\mathrm{ph}},
$$


TABLE II. Differences in the Madelung energy $\left(\Delta E_{M}\right)$, repulsive energy $\left(\Delta E_{R}\right)$, van der Waals energy $\left(\Delta E_{\mathrm{vdW}}\right)$, and polarization energy $\left(E_{p}\right)$ before and after electron-phonon interactions are considered. These differences are denoted by the subscript of $a-b$. The increases in the lattice energies due to formation of dimers, $E_{D}$, the electron-phonon interaction energies, $E_{e \text {-ph }}$, the electron-phonon coupling constants between $\mathrm{V}^{4+}$ ions, $\lambda$, and the relevant elastic constants, $c$, are also tabulated. Every term is obtained per two $\mathrm{VO}_{2}$ molecules as a function of the parameter $u$.

\begin{tabular}{lccccc}
\hline \hline \multicolumn{1}{c}{$u$} & 0.295 & 0.300 & 0.305 & 0.310 & 0.315 \\
\hline$\left(\Delta E_{M}\right)_{a-b}(\mathrm{eV})$ & -0.13 & -0.20 & -0.26 & -0.29 & -0.33 \\
$\left(\Delta E_{R}\right)_{a-b}(\mathrm{eV})$ & 0.00 & 0.00 & 0.00 & -0.01 & -0.01 \\
$\left(\Delta E_{\mathrm{vdW}}\right)_{a-b}(\mathrm{eV})$ & 0.00 & 0.00 & 0.01 & 0.01 & 0.02 \\
$\left(E_{p}\right)_{a-b}(\mathrm{eV})$ & 0.08 & 0.06 & 0.02 & -0.02 & -0.06 \\
$E_{D}(\mathrm{eV})$ & 0.05 & 0.14 & 0.23 & 0.31 & 0.38 \\
$E_{e-\mathrm{ph}}(\mathrm{eV})$ & -0.19 & -0.43 & -0.67 & -0.88 & -1.08 \\
$\lambda(\mathrm{eV} / \AA)$ & -17.9 & -27.7 & -35.2 & -40.2 & -44.4 \\
$c\left(\mathrm{eV} / \AA^{2}\right)$ & 1113 & 1322 & 1414 & 1422 & 1409 \\
\hline \hline
\end{tabular}

$$
\frac{1}{2} c \sum_{i \neq j} x_{i j}^{2}=\left(E_{\text {Relax }}\right)_{b}-\left(E_{\text {Relax }}\right)_{a}=E_{D} .
$$

\section{Discrete-variational $X \boldsymbol{\alpha}$ cluster method}

Based upon the Mott-Hubbard regime, the band gap between the upper and the lower Hubbard bands, $E_{G}$, is generally defined as $^{26,27}$

$$
E_{G}=U-\frac{1}{2}\left(B_{1}+B_{2}\right)+\frac{\lambda^{2}}{2 c},
$$

where $U, B_{1}$, and $B_{2}$ are the intersite repulsive Coulombic interaction, the upper Hubbard bandwidth and the lower one, respectively. In the insulating phase, the empty $\pi^{*}$ and $d_{\|}$ bands belong to the upper Hubbard band, and the occupied narrow $d_{\|}$band is the lower one. Then " $U$ " quantifies the degree of the contribution of the correlation effect for $d$ electrons whereas " $\lambda{ }^{2} / 2 c$ " measures that of the electron-phonon interaction effect which is involved only in the calculation relevant to the occupied narrow $d_{\|}$band in the insulating phase because $\mathrm{V}^{4+}-\mathrm{V}^{4+}$ dimers stabilize the lower $d_{\|}$band. Every term in Eq. (12) is calculated by using the DV-X $\alpha$ cluster method, except the last one which is to be evaluated by the shell model. Therefore, the combination of the cluster method and the shell model is the indispensable means for the energy calculation relevant to the MI transition in $\mathrm{VO}_{2}$.

In order to calculate the electronic structures in both the metallic and the insulating phases using an $a b$ initio molecular-orbital method, the $\left[\mathrm{V}_{2} \mathrm{O}_{10}\right]^{-12}$ clusters were employed in the calculations. All ions were arranged in the tetragonal phase positions in the metallic phase whereas the ionic positions within the cluster in the insulating phase were the ones in the initial structure determined by the periodic shell model. These clusters involve 138 electrons and the average net charges of vanadium and oxygen ions are $+2.05 e$ and $-1.61 e$, respectively. Furthermore, the numerical atomic basis functions, $\mathrm{V} ; 1 s \sim 4 p$ and $\mathrm{O} ; 1 s \sim 2 p$, were used to expand the molecular orbitals. These basis functions are the Slater-type orbital basis sets obtained from the short Herman-Skillman programs. ${ }^{50}$
The calculations used the DV-X $\alpha$ calculation program constructed by Satoko ${ }^{15-19}$ for MS-DOS personal computers. [The source program is provided from the internet (http://www-cms.phys.s.u-tokyo.ac.jp/ $\sim \mathrm{cms} /$ Welcome.html).] The DV-X $\alpha$ method based upon a self-consistent-field Hartree-Fock-Slater (HFS) one-electron model ${ }^{59}$ and a selfconsistent-charge procedure ${ }^{14}$ is one of the most useful techniques for approximately solving the HFS molecular equation. In fact, the electronic structures in several ionic crystals ${ }^{16-20}$ were investigated by this method. Since Adachi, Tsukada, and Satoko ${ }^{16}$ reported the details of the computational treatments of the DV- $X \alpha$ method with the exchangescaling parameter of $\alpha=0.7$ for the Slater $X \alpha$ exchangecorrelation potential, the present calculation has followed them.

The state densities of the clusters are obtained by the overlap of the Gaussian functions with the wide parameter of $0.1 \mathrm{eV}$, which are centered on each of the molecular-orbital energy levels. ${ }^{17}$ It should be emphasized that the present theoretical treatment can formulate the very slight local lattice distortion induced by the electron-phonon interaction using the Aderson's attractive potential ${ }^{23,24}$ and the threedimensional periodic shell model. ${ }^{29}$

\section{RESULTS AND DISCUSSION}

Table II tabulates energy terms per two $\mathrm{VO}_{2}$ molecules calculated by the periodic shell model when $u=0.305$. The energy units per two $\mathrm{VO}_{2}$ molecules are employed because every energy was evaluated per $\mathrm{V}^{4+}-\mathrm{V}^{4+}$ dimer. The subscript of $a-b$ refers to the energy difference between the lattice containing the even distribution of $\mathrm{V}^{4+}$ ions and that in which $\mathrm{V}^{4+}-\mathrm{V}^{4+}$ dimers are formed. As shown in Eq. (10), the difference in the total lattice energy, $\left(E_{T}\right)_{a-b}$, is $\lambda^{2} / 2 c$ and this is also obtained by the specific sum of $\left(\Delta E_{M}\right)_{a-b}$ $+\left(\Delta E_{R}\right)_{a-b}+\left(\Delta E_{\mathrm{vdW}}\right)_{a-b}+\left(E_{p}\right)_{a-b}-E_{e-\mathrm{ph}}$. The energy terms at $u=0.305$ in Table II yields $\left(E_{T}\right)_{a-b}=0.44 \mathrm{eV}$. This agrees with the value of $\lambda^{2} / 2 c$ which is evaluated by the numerical substitutions of $\lambda$ and $c$ in Table II.

As is well known, the electrons on a $\mathrm{V}^{4+}$ site are stabilized by the electron-phonon interactions which draw the neighboring $\mathrm{V}^{4+}$ ions to the electrons. ${ }^{25}$ Then the spacing of 
the $\mathrm{V}^{4+}-\mathrm{V}^{4+}$ pair decreases, that is, $x_{i j}$ in Eq. (1) is negative. The stability of $\mathrm{V}^{4+}-\mathrm{V}^{4+}$ dimers requires a negative value for $E_{e \text {-ph }}$ and accordingly $\lambda$ must be negative. The energy values in Table II are surely subject to this requirement. Consequently the periodic shell model provides the direct evidence that $\mathrm{V}^{4+}-\mathrm{V}^{4+}$ pairs are stabilized and form the dimers in the low-temperature insulating phase, that is, this phase is stable.

Though the calculation employed $u=0.305$, this is the rutile parameter in the high-temperature metallic phase and there are no assurances that $u=0.305$ holds in the insulating phase. Such a situation demands us to repeat the calculations as a parametric function of $u$. As shown in Table II, this parameter was changed from $u=0.295$ to 0.315 at 0.05 increments. This treatment involves the alternation of the initial ionic positions. The energy terms recalculated with the new parameters of $u$ are also summarized in Table II and the stability of the dimers is assured at each value for $u$. The results in Table II indicate that an increase of $u$ requires an increase in the contribution of the electron-phonon interactions because every parameter relevant to the electronphonon interactions; i.e., $E_{D},\left|E_{e-\mathrm{ph}}\right|,|\lambda|$, and $c$, increases with increasing $u$.

In order to investigate the transformation of the electronic structure due to the MI transition in $\mathrm{VO}_{2}$, we carried out the DV-X $\alpha$ cluster calculation. Figures 1(a) and 1(b) show the structures of the $\left[\mathrm{V}_{2} \mathrm{O}_{10}\right]^{-12}$ clusters which were used in the calculations for the metallic and the insulating phases, respectively. In the Mott-Hubbard regime, the energy gap between the upper and the lower Hubbard bands is generally formulated by Eq. (12). Each term in the right-hand side of this equation has been calculated using the cluster method and the shell model, and then these terms yield the band gap, $E_{G}$. Table III tabulates the magnitudes of $B_{1}, B_{2}, U, \lambda^{2} / 2 c$, and $E_{G}$ against $u$. As $u$ increases, the electron-phonon interaction effect represented by $\lambda^{2} / 2 c$ becomes strong considerably as described just above, while the electron correlation effect quantified by $U$ weakens. This is due to the fact that an increase in the $\mathrm{V}^{4+}-\mathrm{V}^{4+}$ separation in the initial state weakens the $d$ - $d$ Coulombic repulsive interaction but enhances the electron-phonon interactions which require a large value for the change in the $\mathrm{V}^{4+}-\mathrm{V}^{4+}$ separation, i.e., $x_{i j}$ in Eq. (1). However, the total value of $U$ and $\lambda^{2} / 2 c$ is nearly constant. Both of $B_{1}$ and $B_{2}$ do not change so much even if $u$ increases. Consequently the band gap is nearly independent of $u$ as shown in Table III, i.e., $E_{G} \cong 0.2 \mathrm{eV}$. Figures 2 (a) and 2(b) illustrate the resultant electronic structures in the metallic and the insulating phases, which are constructed using the energy terms in Tables II and III when $u=0.305$.

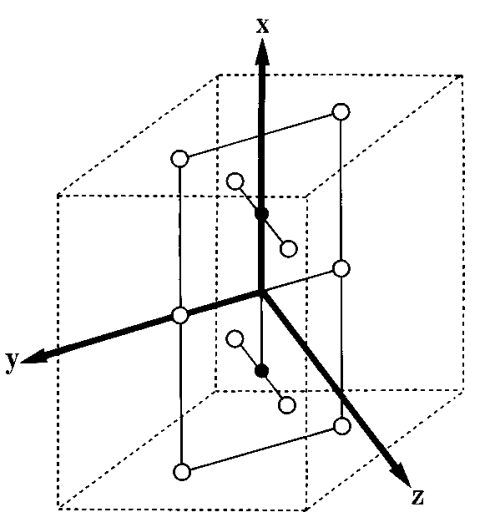

(a)

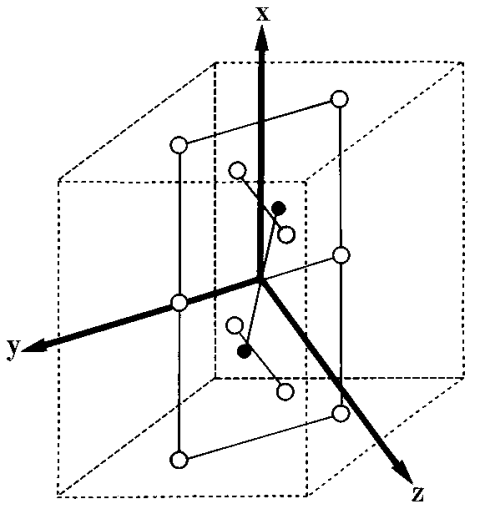

(b)

FIG. 1. Geometric structures of the clusters used in the DV-X $\alpha$ cluster method; (a) the $\left[\mathrm{V}_{2} \mathrm{O}_{10}\right]^{-12}$ cluster in high-temperature metallic phase and (b) that in low-temperature insulating phase, where open circles represent $\mathrm{O}^{2-}$ ions and closed ones $\mathrm{V}^{4+}$. All ions in the metallic phase are arranged in the tetragonal phase positions, while the ionic positions in the initial structure determined by the periodic shell model are employed in the insulating phase.

The most important point in the present results is that the $\pi^{*}$ band and $d_{\|}$band overlap at the Fermi level in the hightemperature metallic phase [Fig. 2(a)], but there is obviously an energy gap between the splitted $d_{\|}$bands, the upper one of which overlaps the $\pi^{*}$ band in the low-temperature insulating phase, as shown in Fig. 2(b). This transformation of the band structure is therefore the theoretical direct evidence for the MI transition in $\mathrm{VO}_{2}$. It should be emphasized that this evidence could not be available if the term of $\lambda^{2} / 2 c$ due to the electron-phonon interactions were not included in the calculations. Consequently, the theoretical description on the MI transition in $\mathrm{VO}_{2}$ requires the combination of the ab initio molecular-orbital method and the periodic shell model.

TABLE III. Upper Hubbard bandwidths, $B_{1}$, lower Hubbard bandwidths, $B_{2}$, intersite repulsive Coulombic interactions, $U$, magnitudes for $\lambda^{2} / 2 c$ which comes from the Anderson's attractive potential, and band gaps, $E_{G}$ against $u$. Every term is in units of $\mathrm{eV}$.

\begin{tabular}{lccccc}
\hline \hline$u$ & 0.295 & 0.300 & 0.305 & 0.310 & 0.315 \\
\hline$B_{1}$ & 1.23 & 1.26 & 1.29 & 1.29 & 1.29 \\
$B_{2}$ & 0.63 & 0.63 & 0.63 & 0.63 & 0.63 \\
$U$ & 1.00 & 0.86 & 0.73 & 0.59 & 0.48 \\
$\lambda^{2} / 2 c$ & 0.14 & 0.29 & 0.44 & 0.57 & 0.70 \\
$E_{G}$ & 0.21 & 0.20 & 0.21 & 0.20 & 0.22 \\
\hline \hline
\end{tabular}


(a)
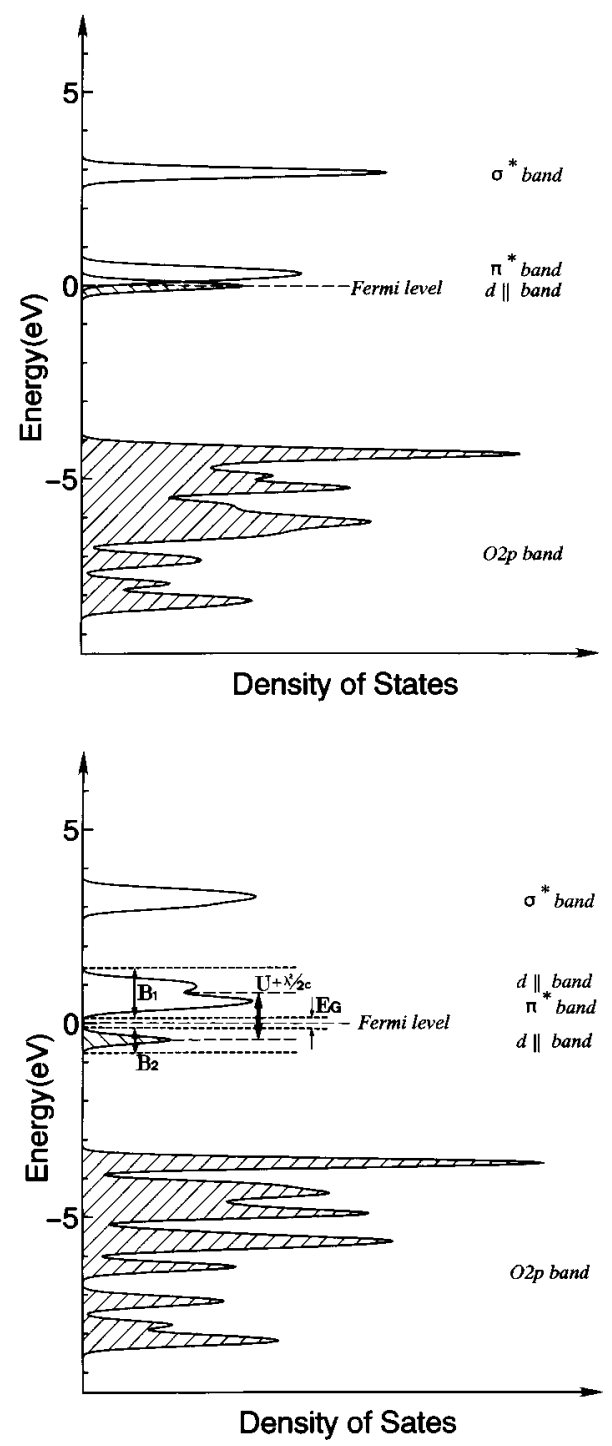

FIG. 2. Electronic structures of $\mathrm{VO}_{2}$ near the Fermi level when $u=0.305$; (a) high-temperature metallic phase and (b) lowtemperature insulating phase. The electronic structure of the metal phase was obtained by the DV-X $\alpha$ cluster method, while that in the insulating phase by the combination of the DV-X $\alpha$ cluster method and the periodic shell model. The occupied bands are shaded. The Fermi energy is taken as the standard level.

The band gap calculated here is smaller than that estimated by the sum of the energy difference between the Fermi level and the top of the $d_{\|}$lower band obtained in the UPS experiment ${ }^{7}$ and the energy difference between the bottom of the $\pi^{*}$ band and the Fermi level estimated from the reflectance measurement, ${ }^{7}$ i.e., $0.7 \mathrm{eV}$. It is interesting that Paquet and Leroux-Hugon ${ }^{22}$ also estimated $E_{G}=0.22 \mathrm{eV}$ taking account of both the electron correlation effect and the electronlattice interactions in their calculations. Their theoretical treatment on the electron-lattice interactions is however quite different from ours. The disagreement in the band gap like this suggests that not only the experiments which can measure the band gap directly but also a development of a fairly better approximation in the cluster calculation method would be necessary so as to understand the behaviors in this material more properly. However, the origin of opening the gap is interpretable in the present calculation.

Despite the disagreement in the band gap, the theoretical treatment in the present report explains rather well the characteristic transformation of the $d_{\|}$band causing the MI transition. The features in Figs. 2(a) and 2(b) are in good agreement with the Goodenough's proposal. ${ }^{6}$ In the metallic phase, $\mathrm{VO}_{2}$ has the tetragonal rutile structure so that the fivefold-degenerate $d$ levels are first split into doubly degenerate $e_{g}$ levels and triply degenerate $t_{2 g}$ levels. The $e_{g}$ levels correspond to the $\sigma^{*}$ band because of a strong hybridization with the $p \sigma$ orbitals, and the $t_{2 g}$ levels are further split into the $d_{\|}$and $\pi^{*}$ levels by the orthorhombic component of the tetragonal crystal field. Therefore, the $\pi^{*}$ band overlaps the half-filled $d_{\|}$band at the lowest energies around the Fermi level as shown in Fig. 2(a). In the insulating phase, $\mathrm{VO}_{2}$ has the monoclinic structure and forms the dimers so that the $\pi^{*}$ band rises in energy beyond the Fermi level and become empty whereas the $d_{\|}$band splits into the empty and the occupied bands as shown in Fig. 2(b).

Caruthers and Kleinman ${ }^{10}$ calculated the band gap of about $0.6 \mathrm{eV}$ using a parametrized tight-binding LCAO band calculation method on the monoclinic crystal structure. They employed the $p-d$ and $d$ - $d$ tight-binding parameters (TBP's) and looked for changes in the TBP's which were consistent with the changes in interatomic distances resulting from the MI transition. Then, their resultant band gap is likely to be an empirical one. Sommers et al. ${ }^{12}$ on the other hand, calculated the $a b$ initio cluster calculation of the electronic $d$ states in $\mathrm{VO}_{2}$, and estimated that the splitting of the $d_{\|}$level was $0.5 \mathrm{eV}$. Their result was obtained in a limit of zero bandwidth so that the band gap cannot be estimated if a bandwidth is included in their calculation. Our theoretical treatment based upon the combination of the cluster method and the shell model seems to be making somewhat remarkable progress.

Furthermore, the relative peak positions of the density of states (DOS) of $\sigma^{*}, \pi^{*}$, and $d_{\|}$bands in the metallic and insulating phases calculated in the present report correspond well to those of $\mathrm{O} 1 s$ absorption spectra obtained by Abbate et al. ${ }^{8}$ Both of their experiment and our calculation yield about $2.7 \mathrm{eV}$ for the energy separation between the DOS peaks of $\sigma^{*}$ and $\pi^{*}$ bands in the metallic and the insulating phases.

In regard to the driving force of the MI transition, Goodenough $^{6}$ was first to emphasize the importance of one shortest V-O separation which is characteristic of a $d-p \pi$ bond in the insulating phase. Zylbersztejn and $\mathrm{Mott}^{27}$ suggested the important role of the $\pi^{*}$ electrons in the MI transition. According to their idea, the electron correlation effect for the $d_{\|}$electrons becomes weak because they are screened by the itinerant $d$ electrons in the $\pi^{*}$ band in the metallic phase. When temperature decreases below $T_{c}$, however, the hybridization between the $\pi^{*}$ and the $p \pi$ orbitals increases mainly because of one shortest V-O separation so that the $\pi^{*}$ band rises in energy beyond the Fermi level and becomes empty. Therefore the correlation energy for the $d_{\|}$electrons becomes enhanced and this correlation effect splits the $d_{\|}$ band into the empty and the occupied bands which should be narrow. According to Paquet and Leroux-Hugon, ${ }^{22}$ the Mott 
transition is the main origin of the MI transition and results in the shortest V-O separation.

Though the $\pi^{*}$ band destabilized by the shortest V-O separation must contribute undoubtedly to the MI transition in $\mathrm{VO}_{2}$ as described before, ${ }^{6,22,27}$ the present study indicates that the $d$ - $d$ electron correlation effect and the electronphonon interaction effect operating in the $\mathrm{V}^{4+}-\mathrm{V}^{4+}$ dimers must participate more directly in the origin of the MI transition. The cluster calculation is then one of the most reliable means so as to account for the transition phenomenon in $\mathrm{VO}_{2}$ if combined with the periodic shell model.

\section{ACKNOWLEDGMENTS}

The authors are very grateful to M. Tsuji and N. Nakamura for useful discussion and assistance in this project. This project was supported by a Grand-in-Aid for Science Research (No. 08650812) from the Ministry of Education, Science and Culture, Japan.
*Author to whom correspondence should be addressed.

${ }^{1}$ S. Westman, Acta Chem. Scand. 15, 217 (1961).

${ }^{2}$ G. Anderson, Acta Chem. Scand. 8, 1599 (1954).

${ }^{3}$ G. Anderson, Acta Chem. Scand. 10, 623 (1956).

${ }^{4}$ B. K. Chakraverty and C. S. Schlenker, J. Phys. (Paris) Colloq. 37, C4-353 (1976).

${ }^{5}$ D. Kaplan, C. Schlenker, and J. J. Since, Philos. Mag. 36, 1275 (1977).

${ }^{6}$ J. Goodenough, Solid State Chem. 3, 490 (1971).

${ }^{7}$ S. Shin, S. Suga, M. Taniguchi, M. Fujisawa, H. Kanzaki, A. Fujimori, H. Daimon, Y. Ueda, K. Kosuge, and S. Kachi, Phys. Rev. B 41, 4993 (1990).

${ }^{8}$ M. Abbate, F. M. de Groot, J. C. Fuggle, Y. J. Ma, C. T. Chen, F. Sette, A. Fujimori, Y. Ueda, and K. Kosuge, Phys. Rev. B 43, 7263 (1991).

${ }^{9}$ Ed. Caruthers, L. Kleinman, and H. I. Zhang, Phys. Rev. B 7, 3753 (1973).

${ }^{10}$ Ed. Caruthers and L. Kleinman, Phys. Rev. B 7, 3760 (1973).

${ }^{11}$ M. Gupta, A. J. Freeman, and D. E. Ellis, Phys. Rev. B 16, 3338 (1977).

${ }^{12}$ C. Sommers, R. de. Groot, D. Kaplan, and A. Zylbersztejn, J. Phys. (Paris) 36, L157 (1975).

${ }^{13}$ C. Sommers and S. Doniach, Solid State Commun. 28, 133 (1978).

${ }^{14}$ A. Rosén, D. E. Ellis, H. Adachi, and F. W. Averill, J. Chem. Phys. 65, 3629 (1976).

${ }^{15}$ A. Zunger and A. J. Freeman, Phys. Rev. B 15, 4716 (1977).

${ }^{16}$ H. Adachi, M. Tsukada, and C. Satoko, J. Phys. Soc. Jpn. 45, 875 (1978).

${ }^{17}$ C. Satoko, M. Tsukada, and H. Adachi, J. Phys. Soc. Jpn. 45, 1333 (1978).

${ }^{18}$ M. Tsukada, C. Satoko, and H. Adachi, J. Phys. Soc. Jpn. 47, 1610 (1979).

${ }^{19}$ M. Tsukada, C. Satoko, and H. Adachi, J. Phys. Soc. Jpn. 48, 200 (1980).

${ }^{20}$ K. Goto, F. Munakata, M. Yamanaka, and H. Adachi, J. Solid State Chem. 119, 76 (1995).

${ }^{21}$ K. H. Johonson and F. C. Smith, Phys. Rev. B 5, 831 (1972).

${ }^{22}$ D. Paquet and P. Leroux-Hugon, Phys. Rev. B 22, 5284 (1980).

${ }^{23}$ P. W. Anderson, Phys. Rev. Lett. 34, 953 (1975).

${ }^{24} \mathrm{P}$. W. Anderson (private communication).

${ }^{25}$ C. Kittel, Introduction to Solid State Physics, 4th ed. (Wiley, New York, 1971), p. 391.

${ }^{26}$ N. F. Mott and L. Friedman, Philos. Mag. 30, 389 (1974).

${ }^{27}$ A. Zylbersztejn and N. F. Mott, Phys. Rev. B 11, 4383 (1975).

${ }^{28}$ G. J. Dienes, D. O. Welch, C. R. Fischer, R. D. Hatcher, O. Lazareth, and M. Samberg, Phys. Rev. B 11, 3060 (1975).
${ }^{29}$ E. Iguchi and H. Nakatsugawa, Phys. Rev. B 51, 10956 (1995).

${ }^{30}$ H. Sawatari, E. Iguchi, and R. J. D. Tilley, J. Phys. Chem. Solids 43, 1147 (1982).

${ }^{31}$ K. Aizawa, E. Iguchi, and R. J. D. Tilley, Proc. R. Soc. London, Ser. A 394, 299 (1984).

${ }^{32}$ E. Iguchi and F. Matsushima, J. Phys. Chem. Solids 47, 45 (1986).

${ }^{33}$ E. Iguchi and T. Yamamoto, J. Phys. Chem. Solids 49, 205 (1988).

${ }^{34}$ E. Iguchi, A. Tamenori, and N. Kubota, Phys. Rev. B 45, 697 (1992).

${ }^{35}$ E. Iguchi and K. Akashi, J. Phys. Soc. Jpn. 61, 3385 (1992).

${ }^{36}$ E. Iguchi, K. J. Lee, and A. Iguchi, J. Phys. Soc. Jpn. 62, 1135 (1993).

${ }^{37}$ E. Iguchi and H. Miyagi, J. Phys. Chem. Solids 54, 403 (1993).

${ }^{38}$ E. Iguchi, T. Hashimoto, and S. Yokoyama, J. Phys. Soc. Jpn. 65, 221 (1996).

${ }^{39}$ W. Paul, Mater. Res. Bull. 5, 691 (1970).

${ }^{40}$ P. P. Ewald, Ann. Phys. (N.Y.) 64, 253 (1921).

${ }^{41}$ W. Van. Gool and A. G. Piken, J. Mater. Sci. 4, 95 (1969).

${ }^{42}$ P. T. Wedepohl, Proc. Phys. Soc. 92, 79 (1967).

${ }^{43}$ P. T. Wedepohl, J. Phys. C 10, 1855 (1977).

${ }^{44}$ P. T. Wedepohl, J. Phys. C 10, 1865 (1977).

${ }^{45}$ R. E. Watson, Phys. Rev. 111, 1108 (1958).

${ }^{46}$ F. Herman and S. Skillman, Atomic Structure Calculations (Prentice Hall, Englewood Cliffs, NJ, 1963), p. 1.

${ }^{47}$ A. R. Ruffa, Phys. Rev. 130, 1412 (1963).

${ }^{48}$ A. R. Ruffa, Phys. Rev. 133, A1418 (1964).

${ }^{49}$ R. A. Parker, Phys. Rev. 124, 1713 (1961).

${ }^{50}$ E. Iguchi, K. Ohtake, T. Yamamoto, and H. Nishikawa, J. Nucl. Mater. 169, 55 (1989).

${ }^{51}$ E. Iguchi and Y. Yonezawa, J. Phys. Chem. Solids 51, 313 (1990).

${ }^{52}$ E. Iguchi, H. Sugimoto, A. Tamenori, and H. Miyagi, J. Solid State Chem. 91, 286 (1991).

${ }^{53}$ E. Iguchi, H. Nishikawa, A. Tamenori, T. Manako, and H. Igarashi, Physica C 179, 393 (1991).

${ }^{54}$ L. Pauling, Proc. R. Soc. London Ser. A 114, 181 (1927).

${ }^{55}$ R. D. Shannon and C. T. Prewitt, Acta Crystallogr. Sec. B 26, 1046 (1970).

${ }^{56}$ B. G. Dick and A. W. Overhauser, Phys. Rev. 112, 90 (1958).

${ }^{57}$ J. S. Shanker, Indian J. Pure. Appl. Phys. 11, 381 (1973).

${ }^{58}$ J. S. Shanker and V. P. Gupta, J. Phys. Chem. Solids 41, 141 (1980).

${ }^{59}$ J. C. Slater, Quantum Theory of Molecules and Solids (McGrawHill, New York, 1974), Vol. 4. 